\title{
Multifractality and critical fluctuations at the Anderson transition
}

\author{
A. D. Mirlin ${ }^{1,2, *}$ and F. Evers ${ }^{1,2}$ \\ ${ }^{1}$ Institut für Nanotechnologie, Forschungszentrum Karlsruhe, 76021 Karlsruhe, Germany \\ ${ }^{2}$ Institut für Theorie der Kondensierten Materie, Universität Karlsruhe, 76128 Karlsruhe, Germany
}

(Received 20 March 2000; revised manuscript received 28 June 2000)

\begin{abstract}
Critical fluctuations of wave functions and energy levels at the Anderson transition are studied for the family of the power-law random banded matrix ensembles. It is shown that the distribution functions of the inverse participation ratios (IPR) $P_{q}$ are scale-invariant at the critical point, with a power-law asymptotic tail. The IPR distribution, the multifractal spectrum, and the level statistics are calculated analytically in the limits of weak and strong couplings, as well as numerically in the full range of couplings.
\end{abstract}

\section{INTRODUCTION}

As is well known, in $d>2$ dimensions a disordered electronic system undergoes, with increasing strength of disorder, a transition from the phase of extended states to that of localized states (Anderson transition). Another important realization of the Anderson critical point is the quantum Hall plateau transition in a two-dimensional (2D) system in strong magnetic field. One of the hallmarks of the metal-insulator transition is represented by strong fluctuations of eigenfunctions. These fluctuations can be characterized by a set of inverse participation ratios (IPR)

$$
P_{q}=\int d^{d} r|\psi(\mathbf{r})|^{2 q} .
$$

The field theory of the Anderson transition is the matrix nonlinear $\sigma$ model, in the replica ${ }^{1}$ or supersymmetric ${ }^{2}$ formulation. In $2+\epsilon$ dimensions with $\epsilon \ll 1$, the transition takes place in the weak-coupling regime, allowing for a systematic renormalization-group $(\mathrm{RG})$ treatment, which yields the critical indices in the form of the $\epsilon$ expansion. In particular, Wegner $^{3}$ found in this way that the IPR show at criticality an anomalous scaling with respect to the system size $L$,

$$
P_{q} \propto L^{-\tau(q)}, \quad \tau(q)=D_{q}(q-1) .
$$

Equation (2) should be contrasted with the behavior of the IPR in a good metal (where eigenfunctions are ergodic), $P_{q}$ $\propto L^{-d(q-1)}$, and, on the other hand, in the insulator (localized eigenfunctions), $P_{q} \propto L^{0}$.

The scaling (2) characterized by an infinite set of critical exponents $D_{q}$ implies that the critical eigenfunction represents a multifractal distribution. ${ }^{4}$ The notion of a multifractal structure was first introduced by Mandelbrot ${ }^{5}$ and was later found relevant in a variety of physical contexts, such as the energy-dissipating set in turbulence, strange attractors in chaotic dynamical systems, and the growth probability distribution in diffusion-limited aggregation; see Ref. 6 for a review. More recently, considerable research interest was attracted by the problem of Dirac fermions in a random vector potential, which allows for an exact calculation of the multifractal spectrum. ${ }^{7-9}$ The multifractal exponents played an important role in recent attempts of identification of the conformal theory describing the quantum Hall plateau transition. ${ }^{10,11}$

During the past decade, multifractality of critical eigenfunctions at the Anderson transition has been a subject of intensive numerical studies; see Refs. 12,13, and references therein. Among all the multifractal dimensions, $D_{2}$ plays the most prominent role, since it determines the spatial dispersion of the diffusion coefficient at the mobility edge. ${ }^{14}$

It should be stressed, however, that Wegner's result (2) refers to an ensemble-averaged IPR. On the other hand, it is well known that in disordered systems, mesoscopic fluctuations from one realization of disorder to another may be very strong. As a result, an average value of some quantity may not provide sufficient information, and one has to speak about the corresponding distribution function. This poses the question of the statistics of the IPR's $P_{q}$ at criticality, which is a central issue of the present paper.

Let us first remind the reader of the existing analytical results concerning the IPR fluctuations. While the direct analytical study of the Anderson transition in 3D is not feasible because of the lack of a small parameter, statistics of energy levels and eigenfunctions in a metallic mesoscopic sample (dimensionless conductance $g \gg 1$ ) can be studied systematically in the framework of the supersymmetry method; see Ref. 15 for a review. Within this approach, the IPR fluctuations were studied recently. ${ }^{16,17,15}$ In particular, the $2 \mathrm{D}$ geometry was considered, which, while not being a true Anderson transition point, shows many features of criticality, in view of the exponentially large value of the localization length. It was found that the distribution function of the IPR $P_{q}$ normalized to its average value $\left\langle P_{q}\right\rangle$ has a scale-invariant form. In particular, the relative variance of this distribution (characterizing its relative width) reads

$$
\frac{\operatorname{var}\left(P_{q}\right)}{\left\langle P_{q}\right\rangle^{2}}=\frac{C q^{2}(q-1)^{2}}{\beta^{2} g^{2}},
$$

where $C \sim 1$ is a numerical coefficient determined by the sample shape (and the boundary conditions), and $\beta=1$ (2) for the case of unbroken (broken) time-reversal symmetry. It is assumed here that the index $q$ is not too large, $q^{2} \ll \beta \pi g$. These findings motivated the conjecture ${ }^{16}$ that the IPR distri- 
bution at criticality has in general a universal form, i.e., that the distribution function $\mathcal{P}\left(P_{q} / P_{q}^{\text {typ }}\right)$ is independent of the size $L$ in the limit $L \rightarrow \infty$. Here $P_{q}^{\text {typ }}$ is a typical value of the IPR, which can be defined, e.g., as a median ${ }^{18}$ of the distribution $\mathcal{P}\left(P_{q}\right)$. Normalization of $P_{q}$ by its average value $\left\langle P_{q}\right\rangle$ (rather than by the typical value $P_{q}^{\text {typ }}$ ) would restrict generality of the statement; see the discussion below. Practically speaking, the conjecture of Ref. 16 is that the distribution function of the IPR logarithm, $\mathcal{P}\left(\ln P_{q}\right)$, simply shifts along the $x$ axis with changing $L$.

The applicability of these results to a generic Anderson transition point has been questioned recently in Ref. 19. Indeed, the 2D metal represents only an "almost critical" point, and the consideration is restricted to the weak disorder limit $g \gg 1$ (weak-coupling regime in the field-theoretical language), while all the realistic metal-insulator transitions (conventional Anderson transition in 3D, quantum Hall transition, etc.) take place in the regime of strong coupling. It was proposed in Ref. 19 (on the basis of numerical simulations for the 3D tight-binding model) that the fractal dimension $D_{2}$ is not a well-defined quantity, but rather shows universal fluctuations characterized by some distribution function $\mathcal{P}\left(D_{2}\right)$ of a width of order unity.

To explore the IPR fluctuations (and also the level statistics, see below) at criticality in the full range from weak to strong coupling, we consider the power-law random banded matrix (PRBM) ensemble. The model is defined ${ }^{20}$ as the ensemble of random Hermitean $N \times N$ matrices $\hat{H}$ (real for $\beta$ $=1$ or complex for $\beta=2$ ). The matrix elements $H_{i j}$ are independently distributed Gaussian variables with zero mean $\left\langle H_{i j}\right\rangle=0$ and the variance

$$
\left\langle\left|H_{i j}\right|^{2}\right\rangle=a^{2}(|i-j|),
$$

where $a(r)$ is given by

$$
a^{2}(r)=\frac{1}{1+(r / b)^{2 \alpha}} .
$$

At $\alpha=1$ the model undergoes an Anderson transition from the localized $(\alpha>1)$ to the delocalized $(\alpha<1)$ phase. We concentrate below on the critical value $\alpha=1$, when $a(r)$ falls down as $a(r) \propto 1 / r$ at $r \gg b$. More precisely, we will study the periodic generalization of Eq. (5),

$$
a^{2}(r)=\left[1+\frac{1}{b^{2}} \frac{\sin ^{2}(\pi r / N)}{(\pi / N)^{2}}\right]^{-1}
$$

(an analog of the periodic boundary conditions), which allows us to get rid of the boundary effects.

In a straightforward interpretation, the PRBM model describes a 1D sample with random long-range hopping, the hopping amplitude decaying as $1 / r^{\alpha}$ with the distance. Also, such an ensemble arises as an effective description in a number of physical contexts, such as the quantum Fermi accelerator, ${ }^{21}$ the delocalization of impurity-induced quasiparticle states in a 2D $d$-wave superconductor, ${ }^{22}$ the scattering by a Coulomb center in an integrable billiard, ${ }^{23}$ the motion of two interacting particles in a 1D random potential, ${ }^{24}$ and the quantum chaos in a billiard with a nonanalytic boundary. ${ }^{25}$ Very recently, a connection between the level statistics of the PRBM model at $b \gg 1$ and the correlations in the Luttinger liquid at finite temperature has been established. $^{26}$

At $\alpha=1$, the PRBM model was found to be critical for an arbitrary value of $b$; it shows all the key features of the Anderson critical point, including multifractality of eigenfunctions and nontrivial spectral compressibility. ${ }^{20,15,45}$ The important property of the ensemble is the existence of the parameter $b$, which labels the critical point: Eqs. (4) and (6) define a whole family of critical theories parametrized by $b .^{27}$ This is in full analogy with the family of the conventional Anderson transition critical points parametrized by the spatial dimensionality $2<d<\infty$. The limit $b \gg 1$ represents a "quasimetallic" regime, with only weakly fractal wave functions, close in their statistical properties to those in a metallic sample. It is analogous to $d=2+\epsilon$ with $\epsilon \ll 1$ and allows for a systematic analytical treatment via the mapping onto a supermatrix $\sigma$ model and the weak-coupling expansion. ${ }^{20,15}$ The opposite limit $b \ll 1$, which we call "quasi-insulating," is characterized by very strongly fluctuating eigenfunctions and corresponds to $d \gg 1$, where the transition takes place in the strong disorder (strong coupling in the field-theoretic language) regime. As we demonstrate below, it is also accessible to an analytical treatment using the RG method introduced by Levitov. ${ }^{28}$ Let us also note a similarity with conformal models proposed recently ${ }^{29,11}$ as candidate theories of the quantum Hall critical point, which are also parametrized by a continuously changing marginal coupling constant.

In this paper, we will combine the analytical study of the eigenfunction and energy level statistics in the two limits $b$ $\gg 1$ and $b \ll 1$ with numerical simulations in the full range of $b$. The feasibility of the systematic analytical treatment of both regimes, weak-coupling and strong-coupling, as well as of the numerical simulations in a broad range of couplings, makes the PRBM ensemble a unique laboratory for studying general features of the Anderson transition.

As has been already mentioned, we will study not only the eigenfunction fluctuations, but also the energy level statistics. It has been understood ${ }^{30-32}$ that a scale-invariant level statistics is an intrinsic feature of the Anderson critical point. In particular, the critical level correlations are characterized by a nontrivial value of the spectral compressibility $0<\chi$ $<1$ (intermediate between $\chi=0$ in a metal and $\chi=1$ in an insulator). ${ }^{30,33,34}$ While the level correlation function itself is shape-dependent, the value of $\chi$ is a fully universal attribute of the critical theory (like critical indices).

Supplementing again the analytical study at $b \gg 1$ and $b$ $\ll 1$ by numerical simulations, we are able to calculate the two-level correlation function $R_{2}(s)$ and the spectral compressibility $\chi$ in the whole range of $b$. Our interest in the critical spectral statistics was additionally motivated by a recent paper, ${ }^{35}$ where it was argued that the following exact relation between $\chi$ and $D_{2}$ holds:

$$
\chi=\frac{d-D_{2}}{2 d} .
$$

According to Eq. (7), the spectral compressibility should tend to $\frac{1}{2}$ in the limit $D_{2} \rightarrow 0$ (very sparse multifractal), and not to the Poisson value $\chi=1$. The derivation of Eq. (7) is 
based, however, on a certain assumption of the decoupling of the energy level and eigenfunction correlations, which is difficult to verify directly. It is important, therefore, to check the validity of the result (7), and such an opportunity is provided by the PRBM model.

The structure of the paper is as follows. Section II is devoted to the IPR statistics and the multifractal spectrum of the PRBM model. In Sec. III, we study the two-level correlation function and the spectral compressibility. Section IV summarizes our findings. Some of the results of this work have been presented in a brief form in Ref. 36 .

\section{MULTIFRACTAL SPECTRUM AND THE IPR STATISTICS}

We find it convenient to organize this section in the following way. We begin by formulating in Sec. II A our main results concerning the IPR statistics. Then we present, in Sec. II B and Sec. II C, the analytical calculations in the limits of $b \ll 1$ and $b \gg 1$, respectively. The numerical simulations (which have been performed for $\beta=1$ ) fully support the analytical findings, ascertaining that the approximations made in the RG treatment are justified. Also, they allow us to explore the intermediate range of $b \sim 1$. A discussion of finite-size effects playing an important role in the analysis of the scaling of the IPR distribution is given in Sec. II D.

\section{A. General considerations and a summary of the results}

For further needs we define two sets of fractal exponents characterizing the scaling of the average IPR $\left\langle P_{q}\right\rangle$ and of the typical IPR $P_{q}^{\text {typ }}$, respectively:

$$
\begin{array}{ll}
\left\langle P_{q}\right\rangle \propto N^{-\tilde{\tau}(q)}, & \tilde{\tau}(q) \equiv \widetilde{D}_{q}(q-1) ; \\
P_{q}^{\mathrm{typ}} \propto N^{-\tau(q)}, & \tau(q) \equiv D_{q}(q-1) .
\end{array}
$$

Note that we consider $q>0$ only; negative $q$ are outside the range of applicability of our analytical methods. As has been already mentioned, $P_{q}^{\text {typ }}$ can be defined as a median of the distribution $\mathcal{P}\left(P_{q}\right){ }^{18}$ an alternative definition can be $P_{q}^{\text {typ }}$ $=\exp \left\langle\ln P_{q}\right\rangle$. Obviously, information about the IPR distribution function $\mathcal{P}\left(P_{q}\right)$ is needed in order to judge whether the exponents $\tilde{\tau}(q)$ and $\tau(q)$ are equal to each other or not. As we will demonstrate below, in the limit of large system size $N$, the distribution $\mathcal{P}\left(P_{q} / P_{q}^{\mathrm{typ}}\right)$ becomes independent of $N$. An important property of this scale-invariant IPR distribution is its power-law "tail" at large $P_{q}$,

$$
\mathcal{P}\left(P_{q} / P_{q}^{\mathrm{typ}}\right) \propto\left(P_{q} / P_{q}^{\mathrm{typ}}\right)^{-1-x_{q},} \quad P_{q} \gg P_{q}^{\mathrm{typ}} .
$$

Of course, the far tail of this distribution becomes increasingly better developed with increasing $N$. In other words, the point where the distribution deviates from its limiting scaleinvariant form moves to infinity as $N$ increases.

It is clear that the relation between $\tau(q)$ and $\tilde{\tau}(q)$ depends crucially on whether the power-law exponent $x_{q}$ is smaller or larger than unity. If $x_{q}>1$, the two definitions of the fractal exponents are identical, $\tau(q)=\tilde{\tau}(q)$. This situation will be shown to occur at not too large values of $q$; in particular, $x_{2}>1$ at any $b$. However, with increasing $q$ the value of $x_{q}$ decreases. Once it drops below unity, the average $\left\langle P_{q}\right\rangle$ starts to be determined by the upper cutoff of the power-law "tail," which depends on the system size. As a result, $\left\langle P_{q}\right\rangle$ shows scaling with an exponent $\tilde{\tau}_{q}$ different from $\tau_{q}$. In this situation, the average value $\left\langle P_{q}\right\rangle$ is not representative and is determined by rare realizations of disorder.

The connection between $\tilde{\tau}(q)$ and $\tau(q)$ in the regime $x_{q}$ $<1$ can be elucidated best via introducing the singularity spectrum $f(\alpha)$, which is the conventional way of analyzing multifractal distributions. ${ }^{6}$ To this end, let us note that the average IPR's $\left\langle P_{q}\right\rangle$ are (up to a multiplication by $N$ ) the moments of the distribution $\mathcal{P}\left(\left|\psi^{2}\right|\right)$ of the eigenfunction intensities. The behavior (8) of the moments corresponds to the intensity distribution function of the form

$$
\mathcal{P}\left(\left|\psi^{2}\right|\right) \sim \frac{1}{\left|\psi^{2}\right|} N^{-1+f\left(-\ln \left|\psi^{2}\right| / \ln N\right)} .
$$

Indeed, calculating the moments $\left\langle\left|\psi^{2 q}\right|\right\rangle$ with the distribution function (11), one finds

$$
\left\langle P_{q}\right\rangle=N\left\langle\left|\psi^{2 q}\right|\right\rangle \sim \int d \alpha N^{-q \alpha+f(\alpha)},
$$

where we have introduced $\alpha=-\ln \left|\psi^{2}\right| / \ln N$. Evaluation of the integral by the saddle-point method reproduces the result (8), with the exponent $\tilde{\tau}(q)$ related to the singularity spectrum $f(\alpha)$ via the Legendre transform

$$
\tilde{\tau}(q)=q \alpha-f(\alpha) ; \quad q=f^{\prime}(\alpha) .
$$

It is not difficult to see that the condition $x_{q}=1$ is equivalent to $f(\alpha)=0$. Indeed, both conditions $x_{q}<1$ and $f(\alpha)<0$ characterize the situation when the average value $\left\langle P_{q}\right\rangle$ is not representative and is determined by rare realizations of disorder. On a more formal level, this can be derived from the formula relating $x_{q}$ and the fractal exponents, see Eq. (16) below and Sec. II C.

We further denote the value of $\alpha$ determined by $f(\alpha)$ $=0$ as $\alpha_{-}$, and the corresponding value of $q$ as $q_{c}$ (clearly, both $\alpha_{-}$and $q_{c}$ depend on $b$ ). The value of $\tau_{q}$ in the region $q>q_{c}$ can be found by observing that $P_{q}^{\text {typ }}$ can be written in a form similar to Eq. (12),

$$
P_{q}^{\mathrm{typ}} \sim \int_{f(\alpha) \geqslant 0} d \alpha N^{-q \alpha+f(\alpha)} .
$$

The restriction on the integration range removes from consideration the rare events of such large values of $|\psi|^{2}$ which can be found only in a small fraction $\left[\sim N^{f(\alpha)}\right.$ with $f(\alpha)$ $<0$ ] of all eigenfunctions. Since for $q>q_{c}$ the saddle point $\alpha<\alpha_{-}$is outside the integration domain, the integral (14) is determined in this case by the boundary $\alpha_{-}$of the integration range, yielding (see a related discussion in Ref. 8)

$$
\tau(q)=q \alpha_{-}, \quad q>q_{c} .
$$

The value of the power-law-tail index $x_{q}$ is related to the fractal exponents as follows:

$$
x_{q} \tau(q)=\tilde{\tau}\left(q x_{q}\right) .
$$


To be precise, we were able to derive Eq. (16) for all $q$ in the limit $b \ll 1$, as well as for integer values of $x_{q}=1,2, \ldots$ at arbitrary $b$. We expect, however, that this relation is generally valid.

According to what has been said above, the curve $q_{c}(b)$ separates the regions with the two different types of multifractal behavior: at $q<q_{c}(b)$ we have $x_{q}>1$ and $\tau(q)$ $=\tilde{\tau}(q)$, while at $q>q_{c}(b)$ the tail index $x_{q}<1$ and $\tau(q)$ is different from $\tilde{\tau}(q)$ and given by Eq. (15). We have calculated the asymptotic form of the "phase boundary" $q_{c}(b)$ in both limits $b \gg 1$ and $b \ll 1$,

$$
q_{c}(b) \simeq \begin{cases}(2 \pi \beta b)^{1 / 2}, & b \gg 1 \\ 2.4056, & b \ll 1 .\end{cases}
$$

Notice that $q=2$ always belongs to the low- $q$ phase, i.e., $\tau(2)=\tilde{\tau}(2)$ for all $b$. For $q>q_{c}(b)$ we find from Eqs. (15) and (16) that $x_{q}=q_{c}(b) / q$, while in the opposite regime $q$ $<q_{c}(b)$ the value of $x_{q}$ is determined by the form of the function $\tilde{\tau}(q)$. In particular, at $b \gg 1$ we have

$$
\widetilde{\tau}(q) \equiv(q-1) \widetilde{D}_{q} \simeq(q-1)(1-q / 2 \beta \pi b),
$$

yielding $x_{q} \simeq 2 \beta \pi b / q^{2}$ for $q<(2 \beta \pi b)^{1 / 2}$. In the other limit, $b \ll 1$, the function $\tilde{\tau}(q)$ has a somewhat more complicated form,

$$
\tilde{\tau}(q) \simeq \frac{4 b}{\sqrt{\pi}} \frac{\Gamma(q-1 / 2)}{\Gamma(q-1)} \times \begin{cases}1, & \beta=1 \\ \pi / 2 \sqrt{2}, & \beta=2,\end{cases}
$$

and Eq. (16) does not seem to have an analytical solution for $x_{q}$. However, for the particularly important case $q=2$, we find $x_{2}=\frac{3}{2}$, while all higher integer $q=3,4, \ldots$ are already above the phase boundary $q_{c}(b \ll 1) \simeq 2.4$.

\section{B. Regime $b \gg 1$}

The quasimetallic regime $b \gg 1$ can be studied via the mapping onto the supermatrix $\sigma$ model. ${ }^{20,15}$ The $\sigma$-model action in momentum space has the form

$$
S[Q]=\beta \operatorname{Str}\left[-\frac{1}{t} \int \frac{d k}{2 \pi}|k| Q_{k} Q_{-k}-\frac{i \pi \nu \omega}{4} Q_{0} \Lambda\right],
$$

where $Q_{k}=\Sigma_{r} e^{i k r} Q(r)$ and $Q(r)$ is a $4 \times 4(\beta=2)$ or 8 $\times 8 \quad(\beta=1)$ supermatrix field constrained by $Q^{2}(r)=1$ and belonging to a coset space with the origin $\Lambda=\operatorname{diag}(\mathbf{1},-\mathbf{1})$. Furthermore, $\operatorname{Str}$ denotes the supertrace, $\nu$ is the density of states given by the Wigner semicircle law

$$
\nu(E)=\frac{1}{2 \pi^{2} b}\left(4 \pi b-E^{2}\right)^{1 / 2}, \quad|E|<2 \sqrt{\pi b},
$$

and $t \ll 1$ is the coupling constant,

$$
\frac{1}{t}=\frac{\pi}{4}(\pi \nu)^{2} b^{2}=\frac{b}{4}\left(1-\frac{E^{2}}{4 \pi b}\right) .
$$

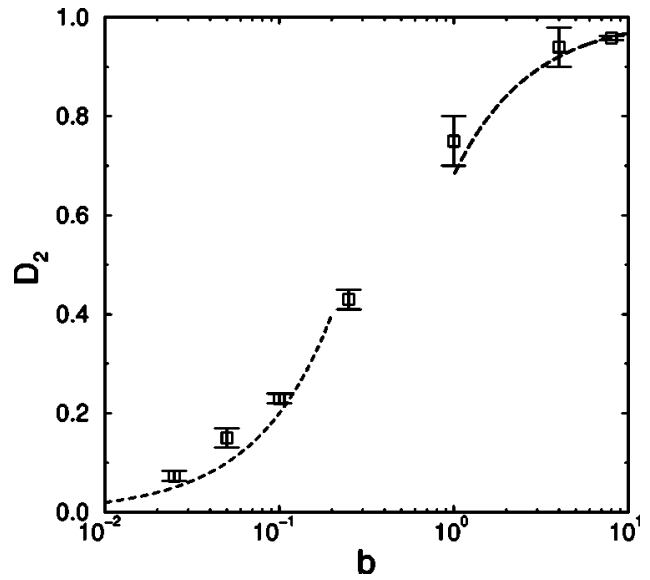

FIG. 1. Fractal dimension $D_{2}$ as a function of the parameter $b$ of the PRBM ensemble. The data points are the results of the numerical simulations, while the lines represent the $b \gg 1$ and $b \ll 1$ analytical asymptotics, $D_{2}=1-1 / \pi b\left[\right.$ Eq. (23)] and $D_{2}=2 b$ [Eq. (49)].

For a system of finite size $N$ with the periodic generalization (6) of the $1 / r$ decay law of $a(r)$, the $k$ integration in Eq. (20) is replaced by summation in the usual way:

$$
\int \frac{d k}{2 \pi} \rightarrow \frac{1}{N}{ }_{k=2 \pi n / N ;} \sum_{n=0, \pm 1, \pm 2, \ldots} .
$$

The eigenfunction statistics can now be studied via the same methods as for conventional metallic samples. The main difference between the action (6) and that of the diffusive $\sigma$ model is in the replacement of the diffusion operator $(\pi \nu / 8) D k^{2}$ by $(1 / t)|k|$. Consequently, all calculations within the weak-coupling expansion of the $\sigma$ model are generalized to the PRBM case by substituting $\Pi(k)=t / 8|k|$ for the diffusion propagator $\Pi(k)=1 / \pi \nu D k^{2}$. In particular, calculating the average IPR $\left\langle P_{q}\right\rangle$, one finds the following result for the fractal dimensions $\widetilde{D}_{q}:{ }^{20,15}$

$$
\widetilde{D}_{q} \simeq 1-q \frac{t}{8 \pi \beta}, \quad q<\frac{4 \pi \beta}{t} .
$$

Figure 1 shows that this result is in good agreement with numerical simulations.

Similar to the case of 2D (Ref. 37) or $(2+\epsilon)$ dimensions, ${ }^{3}$ Eq. (23) describes weak multifractality: the deviation of the fractal exponents from the "normal" value $\widetilde{D}_{q}=d=1$ (characteristic for homogenously spread, "metallic" wave functions) is proportional to the small parameter $t$. The Legendre transform of $\tilde{\tau}(q)=(q-1) \widetilde{D}_{q}$ yields the singularity spectrum (for definiteness, we concentrate on the band center $E=0$, where $t=4 / b$ )

$$
f(\alpha) \simeq 1-\frac{\left(\alpha_{0}-\alpha\right)^{2}}{4\left(\alpha_{0}-1\right)}, \quad \alpha_{0}=1+\frac{1}{2 \beta \pi b},
$$

which crosses the $x$ axis at the point

$$
\alpha_{-} \simeq\left[1-\frac{1}{(2 \beta \pi b)^{1 / 2}}\right]^{2},
$$




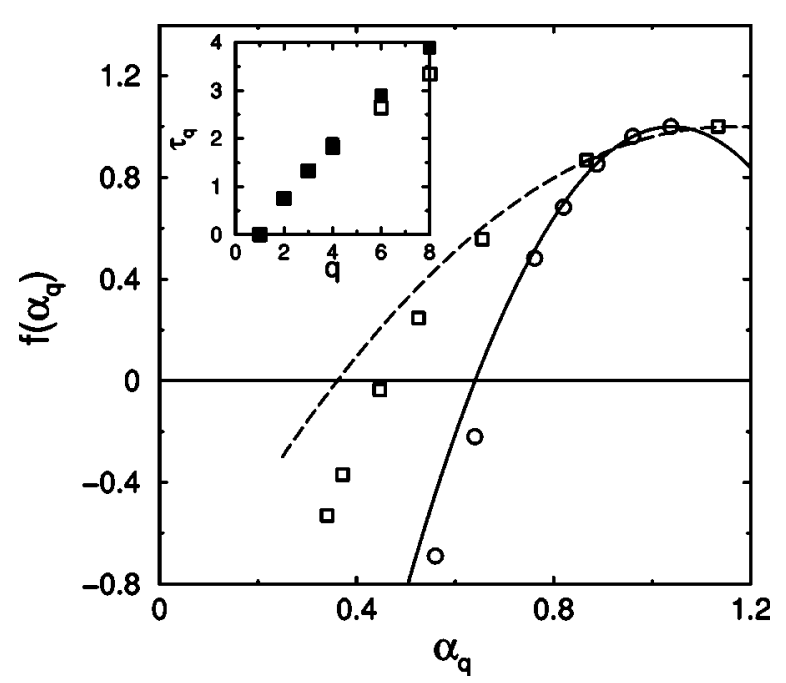

FIG. 2. Multifractal spectrum $f(\alpha)$ for $b=1$ ( $\square)$ and $b=4$ (O). The lines indicate the parabolic approximation Eq. (24). Inset: exponent $\tilde{\tau}(q)(\square)$ and $\tau(q)(\boldsymbol{\square})$ for $b=1$.

corresponding to $q_{c}(b)=(2 \beta \pi b)^{1 / 2}$.

In Fig. 2 we confront our analytical findings with data from numerical simulations. At $b=4$ the parabola represents the numerical data well up to $q \sim 8$. The deviations from the asymptotic (parabolic) form are much more pronounced at $b=1$. These deviations are a precursor of the crossover to the small- $b$ regime (Sec. II C), where the parabolic approximation breaks down completely. The sign of the deviations (downwards) is consistent with the fact that at $b=1 / 2 \pi$ the parabolic approximation would predict $\alpha_{-}=0$, while we expect $\alpha_{-}>0$ for all $b$, in view of the absence of localized states.

Now we turn to the IPR fluctuations, which are found by generalizing the results obtained for metallic samples. ${ }^{16,17,15}$ In particular, the IPR variance is given for $q \ll q_{c}(b)$ by ${ }^{20}$

$$
\begin{aligned}
\frac{\operatorname{var}\left(P_{q}\right)}{\left\langle P_{q}\right\rangle^{2}} & =\frac{2}{\beta^{2}} q^{2}(q-1)^{2} \frac{1}{N^{2}} \sum_{k} \Pi^{2}(k) \\
& =\frac{1}{24 \beta^{2}} \frac{q^{2}(q-1)^{2}}{b^{2}}
\end{aligned}
$$

where the $k$ summation goes over the nonzero harmonics $k$ $=2 \pi j / N$ with $j= \pm 1, \pm 2, \ldots$ Equation (26) is the PRBM counterpart of formula (3) for 2D metallic systems. The higher moments of the IPR distribution were studied by Prigodin and Altshuler ${ }^{17}$ (see also Ref. 15); generalizing these results, we find that the irreducible moments (cumulants) of the order $2 \leqslant n \ll 2 \pi \beta b / q^{2}$ are given by

$$
\begin{aligned}
\frac{\left\langle\left\langle P_{q}^{n}\right\rangle\right\rangle}{\left\langle P_{q}\right\rangle^{n}} & =\frac{(n-1) !}{2}\left[\frac{2}{\beta} q(q-1)\right]^{n} \frac{1}{N^{n}} \sum_{k} \Pi^{n}(k) \\
& =(n-1) !\left(\frac{q(q-1)}{2 \pi \beta b}\right)^{n} \zeta(n),
\end{aligned}
$$

where $\zeta(n)$ is the Riemann $\zeta$ function. Defining in analogy with $^{17}$

$$
\widetilde{P}=\left[\frac{P_{q}}{\left\langle P_{q}\right\rangle}-1\right] \frac{2 \pi \beta b}{q(q-1)},
$$

we have for the cumulants of $\widetilde{P}$

$$
\begin{gathered}
\langle\widetilde{P}\rangle=0, \\
\left\langle\left\langle\widetilde{P}^{n}\right\rangle\right\rangle=(n-1) ! \zeta(n) \equiv K_{n}, \quad n=2,3, \ldots .
\end{gathered}
$$

This allows us to restore the corresponding distribution function:

$$
\begin{aligned}
\mathcal{P}(\widetilde{P}) & =\int_{-\infty}^{\infty} \frac{d s}{2 \pi} e^{i s \widetilde{P}} \exp \left[\sum_{n=2}^{\infty} K_{n} \frac{(-i s)^{n}}{n !}\right] \\
& =\int_{-\infty}^{\infty} \frac{d s}{2 \pi} e^{i s(\widetilde{P}+\mathbf{C})} \Gamma(1+i s) \\
& =e^{-\widetilde{P}-\mathbf{C}} \exp \left(-e^{-\tilde{P}-\mathbf{C}}\right),
\end{aligned}
$$

where $\mathbf{C} \simeq 0.5772$ is the Euler constant. The restriction on $n$ given above (27) implies that Eq. (30) is valid for $P_{q} /\left\langle P_{q}\right\rangle$ $-1 \ll 1$.

The similarity with the 2D metallic regime extends also to the asymptotic behavior of the distribution. Specifically, at $P_{q} /\left\langle P_{q}\right\rangle-1 \sim 1$ the exponential falloff (30) crosses over to a power-law tail (see Ref. 15 for the discussion of this tail in 2D)

$$
\mathcal{P}\left(P_{q}\right) \sim\left(P_{q} /\left\langle P_{q}\right\rangle\right)^{-1-x_{q}} .
$$

To calculate $x_{q}$, we consider the moments

$$
\left\langle P_{q}^{n}\right\rangle=\sum_{r_{1}, \ldots, r_{n}}\left|\psi\left(r_{1}\right)\right|^{2 q} \cdots\left|\psi\left(r_{n}\right)\right|^{2 q} .
$$

For $n$ not too large, the sum is dominated by the main part of the total configuration space, with all points $r_{i}$ lying far from each other, so that $\left\langle P_{q}^{n}\right\rangle \sim\left\langle P_{q}\right\rangle^{n} \sim N^{-n \tau(q)}$. In contrast, for sufficiently large $n$ the integral is dominated by the contribution from $r_{1} \approx r_{2} \approx \cdots \approx r_{n}$, yielding $\left\langle P_{q}^{n}\right\rangle \sim N^{-\tilde{\tau}(n q)}$. Therefore, if

$$
n \tau(q)=\tilde{\tau}(n q),
$$

we have the marginal situation, which implies that $\mathcal{P}\left(P_{q}\right)$ $\propto P_{q}^{-1-n}$. This completes the derivation of Eq. (16) in the range $q<q_{c}(b)$. Using now Eq. (16) in combination with Eq. (23), we find

$$
x_{q}=\frac{2 \pi \beta b}{q^{2}}, \quad q^{2}<2 \pi \beta b .
$$

Note that an analogous consideration in the $2 \mathrm{D}$ case yields $x_{q}=2 \pi \beta g / q^{2}$, in full agreement with the result of the optimum-fluctuation method. ${ }^{15}$

We have therefore presented an explicit calculation of the IPR distribution function at $b \gg 1$ and $q<q_{c}(b)$. The distribution function is scale-invariant and has the form (30) and (28) at $P_{q} /\left\langle P_{q}\right\rangle-1 \ll 1$ and the power-law tail (31) and (34) at $P_{q} /\left\langle P_{q}\right\rangle-1 \gtrsim 1$. Figure 3 shows results of the numerical simulations for the distribution of the IPR's $P_{q}$ with $q=2,4$, and 6 at $b=4$ [the corresponding value of $q_{c}$ being $q_{c}$ 


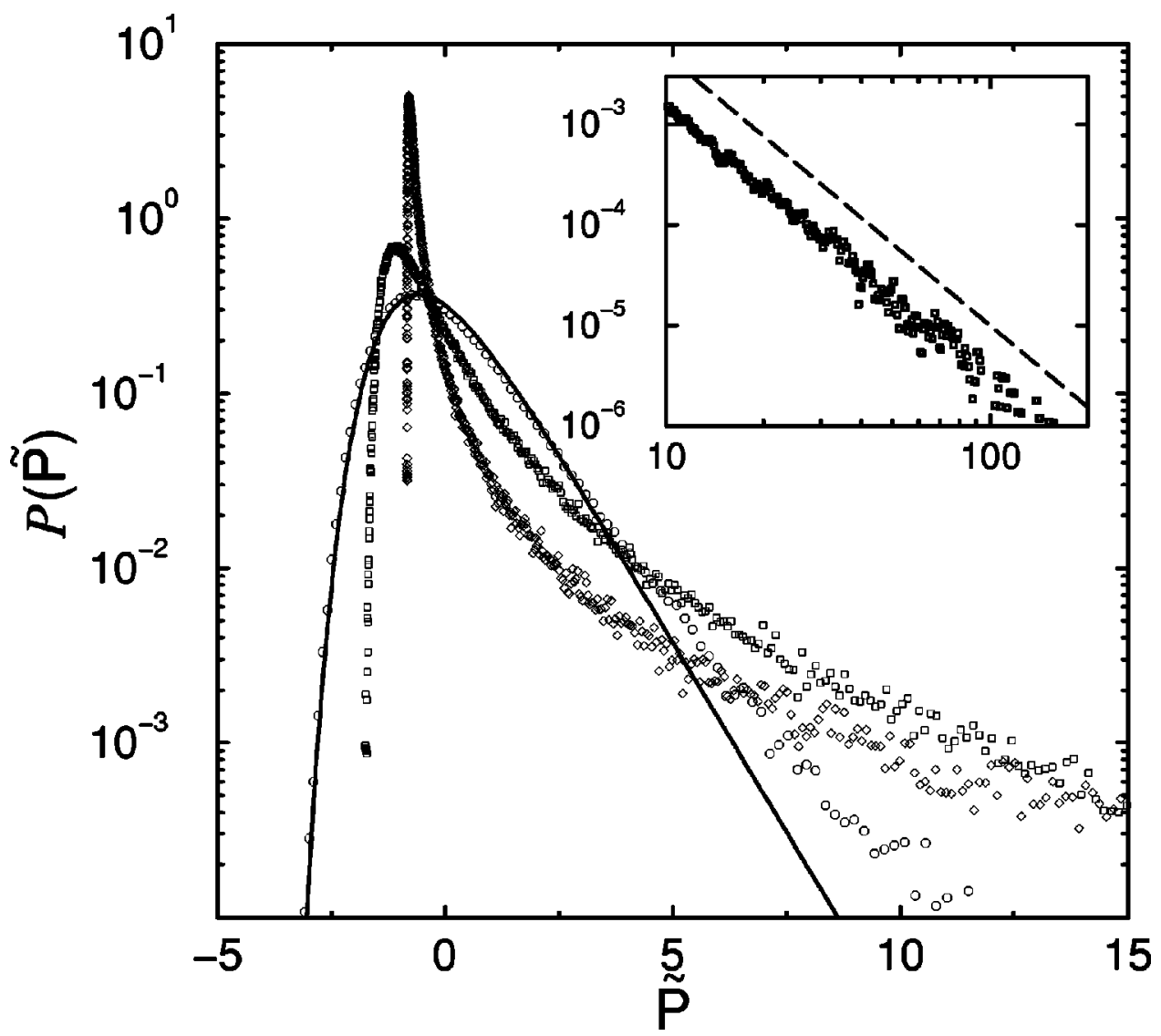

FIG. 3. Distribution function $\mathcal{P}\left(\widetilde{P}_{q}\right)$ at $q=2(\bigcirc), 4(\square)$, and $6(\diamond)$ at $b=4$ for systems of size $N=4096$. The solid line represents the analytical result Eq. (30). The scattering of the data at small values of $\mathcal{P}$ is due to statistical noise. (Number of matrices in the ensemble: 428.) Inset: Asymptotic of $\mathcal{P}\left(\widetilde{P}_{4}\right)$. Dashed line indicates power law with exponent $x_{4}=1.7$.

$=(8 \pi)^{1 / 2} \simeq 5$ ]. It is seen that at $q=2$ the analytical formula (30) nicely describes the "main body" of the distribution, with the upward deviations at large $\widetilde{P}$ indicating the crossover to the power-law tail (31). The asymptotic behavior (31) is outside the reach of our numerical simulations for $q$ $=2$, however, since the condition of its validity $\widetilde{P}$ $\gg 2 \pi \beta / q(q-1) \simeq 12.5$ corresponds to very small values of the distribution function $\mathcal{P}(\widetilde{P}) \ll 10^{-5}$ and its clear resolution would require a much larger statistical ensemble. The situation changes, however, with increasing $q$ (see the data for $q=4$ and 6 in Fig. 3). Equation (30) becomes inapplicable (since the condition of its validity $q \ll q_{c}$ is not met anymore) and the power-law asymptotic behavior (31) becomes clearly seen. In particular, the inset of Fig. 3 shows the tail for $q$ $=4$; the extracted value of the index $x_{4} \simeq 1.7$ is in good agreement with the prediction of the $b \gg 1$ theory, $x_{4}=\pi / 2$.

In conclusion of this subsection, we comment on the notion of the termination of the multifractal spectrum (24), which has been discussed in the literature on disordered Dirac fermions. ${ }^{9}$ It is important to realize that, in the present context, there are two types of such termination, depending on whether one studies $P_{q}^{\text {typ }}$ or $\left\langle P_{q}\right\rangle$. In the former case, the relevant values of $\alpha$ are those where $f(\alpha) \geqslant 0$, so that the singularity spectrum $f(\alpha)$ effectively terminates at $\alpha_{-}$ [which corresponds to $q=q_{c}(b) \simeq(2 \pi \beta b)^{1 / 2}$ ]. For $q$ $>q_{c}(b), \tau(q)$ is given by Eq. (15), so that the fractal exponent $D_{q}=q \alpha_{-} /(q-1)$ saturates as $D_{q} \rightarrow \alpha_{-}$in the limit $q$ $\rightarrow \infty$. In contrast, if the exponent $\tilde{\tau}(q)$ describing the scaling of the average $\left\langle P_{q}\right\rangle$ is studied, then the behavior (23) continues up to $q \approx \pi \beta b$, which corresponds to $\alpha=0$. This type of termination [which takes place at $q$ parametrically much larger than $\left.q_{c}(b)\right]$ has a physically transparent origin: since $\alpha=-\ln \left|\psi^{2}\right| / \ln N$ and in view of the wave-function normalization, the allowed values of $\alpha$ are restricted by $\alpha \geqslant 0$. More detailed discussion of the behavior of $\tilde{\tau}(q)$ in the vicinity of $q=\pi \beta b$ (i.e., the precise form of this termination) is outside the scope of the present paper.

\section{Regime $b \ll 1$}

In the quasi-insulating case $b \ll 1$ the problem can be studied via the renormalization-group method of Levitov. ${ }^{28,38}$ The idea of the method is as follows. One starts from the diagonal part of the matrix $\hat{H}$, each eigenstate being localized on a single site. Then one includes into consideration nondiagonal matrix elements $H_{i j}$ with $d(i, j)=1$, where $d(i, j)$ is the distance between the sites $i$ and $j$ with periodic boundary conditions taken into account,

$$
d(i, j)=\min \{|i-j|, N-|i-j|\} .
$$

Now one argues that most of these matrix elements are essentially irrelevant, since their typical value is $\sim b$, while the energy difference $\left|E_{i}-E_{j}\right|$ is typically of order unity. Only with a small probability $(\sim b)$ is $\left|E_{i}-E_{j}\right|$ also of the order of 
$b$, so that the matrix element mixes strongly the two states. Following Levitov, we will say that these two states are in resonance. In this case one is led to consider a two-level problem

$$
\hat{H}_{\text {two-level }}=\left(\begin{array}{cc}
E_{1} & V \\
V & E_{2}
\end{array}\right) .
$$

The corresponding eigenfunctions and eigenenergies are

$$
\begin{gathered}
\psi^{(+)}=\left(\begin{array}{c}
\cos \theta \\
\sin \theta
\end{array}\right), \quad \psi^{(-)}=\left(\begin{array}{c}
-\sin \theta \\
\cos \theta
\end{array}\right), \\
E_{ \pm}=\frac{E_{1}+E_{2}}{2} \pm|V| \sqrt{1+\tau^{2}}
\end{gathered}
$$

where

$$
\begin{gathered}
\tan \theta=-\tau+\sqrt{1+\tau^{2}}, \\
\tau=\frac{\omega}{2 V}, \quad \omega=E_{1}-E_{2} .
\end{gathered}
$$

In the next RG step the matrix elements $H_{i j}$ with $d(i, j)$ $=2$ are taken into account, then those with $d(i, j)=3$, and so forth until $d(i, j)=N / 2$. Each time a resonance is encountered, the Hamiltonian is reexpressed in terms of the new states. Since the probability of a resonance at a distance $r$ $=d(i, j)$ is $\sim b / r$, the typical scale $r_{2}$ at which a resonance state formed at a scale $r_{1}$ will again be in resonance satisfies

$$
\ln \frac{r_{2}}{r_{1}} \sim \frac{1}{b}
$$

so that $r_{2}$ is much larger than $r_{1}$. Therefore, when considering the resonant two-level system at the scale $r_{2}$, one can treat the $r_{1}$ resonance state as pointlike. Furthermore, it is easy to see that the Gaussian statistics of the matrix element coupling the states on the scale $r_{2}$ is not affected by the transformation to the new basis induced by the $r_{1}$ resonance.

Now we consider the evolution of the IPR distribution with the distance $r$; we will denote the corresponding distribution function as $f\left(P_{q}, r\right)$. When a resonance occurs, two states with IPR's $P_{q}^{(1)}$ and $P_{q}^{(2)}$ are replaced by two new states with the IPR's,

$$
\begin{aligned}
& P_{q}^{(+)}=P_{q}^{(1)} \cos ^{2 q} \theta+P_{q}^{(2)} \sin ^{2 q} \theta, \\
& P_{q}^{(-)}=P_{q}^{(1)} \sin ^{2 q} \theta+P_{q}^{(2)} \cos ^{2 q} \theta .
\end{aligned}
$$

We thus have for real matrices $(\beta=1)$

$$
\begin{aligned}
\frac{\partial}{\partial r} f( & \left.P_{q}, r\right) \\
= & 2 \nu \int_{-\infty}^{\infty} d \omega \int_{-\infty}^{\infty} d V \frac{1}{\sqrt{2 \pi}} \frac{\tilde{r}}{b} e^{-V^{2} \tilde{r}^{2} / 2 b^{2}} \\
& \times\left(-f\left(P_{q}, r\right)+\int d P_{q}^{(1)} d P_{q}^{(2)} f\left(P_{q}^{(1)}, r\right) f\left(P_{q}^{(2)}, r\right)\right.
\end{aligned}
$$

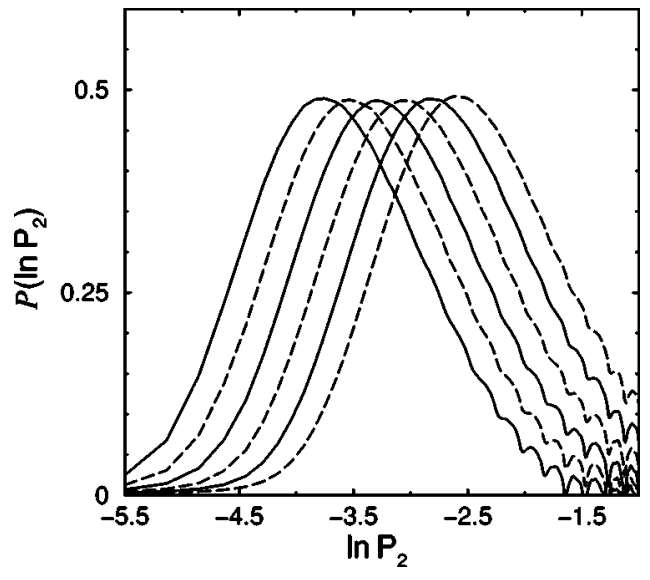

FIG. 4. Flow of the distribution of $\ln P_{2}$ calculated from the kinetic equation (46) at $t=b \ln r=1.2, \ldots, 1.7$ (from right to left). The oscillations near $\ln P_{2}=-1.5$ are numerical artifacts due to rounding errors.

$$
\left.\delta\left(P_{q}-P_{q}^{(1)} \cos ^{2 q} \theta-P_{q}^{(2)} \sin ^{2 q} \theta\right)\right)
$$

where $\nu=(1 / \sqrt{2 \pi}) e^{-E^{2} / 2}$ is the density of states and

$$
\tilde{r}=\frac{N}{\pi} \sin \frac{\pi r}{N} .
$$

The difference between $\tilde{r}$ and $r$ is irrelevant for the present calculation, since the $r$ integral will be of a logarithmic nature and thus dominated by $r \ll N$. However, this difference is important for the calculation of the level correlation function below (Sec. III). Transforming the integration measure according to

$$
d \omega=2 V d \tau, \quad d \tau=-\frac{1}{2 \sin ^{2} \theta \cos ^{2} \theta} d \theta,
$$

calculating the $V$ integral, and specializing on the band center $(E=0)$ for definiteness, we reduce the evolution equation (43) to the form

$$
\begin{aligned}
\frac{\partial}{\partial \ln r} & f\left(P_{q}, r\right) \\
= & \frac{2 b}{\pi} \int_{0}^{\pi / 2} \frac{d \theta}{\sin ^{2} \theta \cos ^{2} \theta} \\
& \times\left(-f\left(P_{q}, r\right)+\int d P_{q}^{(1)} d P_{q}^{(2)} f\left(P_{q}^{(1)}, r\right) f\left(P_{q}^{(2)}, r\right)\right. \\
& \left.\times \delta\left(P_{q}-P_{q}^{(1)} \cos ^{2 q} \theta-P_{q}^{(2)} \sin ^{2 q} \theta\right)\right) .
\end{aligned}
$$

Equation (46) is a kind of kinetic equation (in the fictitious time $t=b \ln r$ ), with the two terms in the large parentheses describing the scattering-out and scattering-in processes, respectively.

Figure 4 shows the results of the numerical integration of Eq. (46) for $q=2$ with the initial condition $f\left(P_{2}\right)=\delta\left(P_{2}\right.$ $-1)$ at $t=0$. It is seen that at sufficiently large $t$ the distribution of $\ln P_{2}$ acquires a limiting form, shifting with $t$ with- 


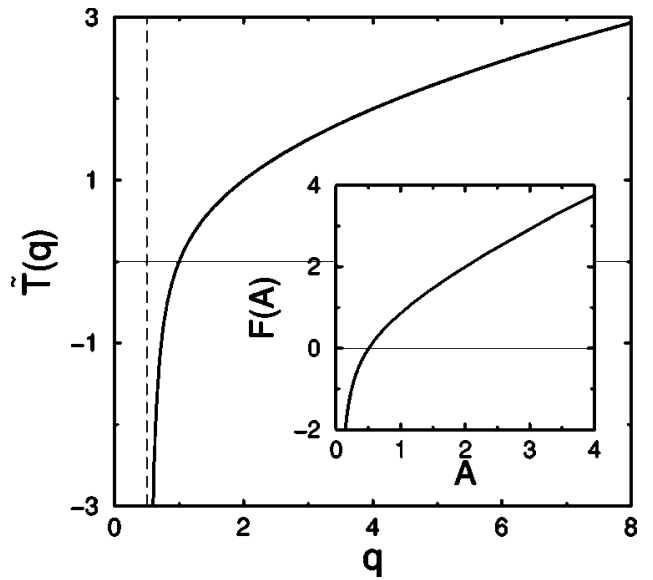

FIG. 5. Universal function $\widetilde{T}(q)$ characterizing the exponents $\tilde{\tau}(q)$ via $\tilde{\tau}(q)=2 b \widetilde{T}(q)$ at $b \ll 1$. Dashed line indicates the pole position. Inset: Legendre transform $F(A)$ describing the multifractal spectrum via $f(\alpha)=2 b F(\alpha / 2 b)$.

out changing its shape. This conclusion of scale invariance of the IPR distribution will be corroborated below by analytical arguments.

Turning to the theoretical analysis, we consider first the average value $\left\langle P_{q}\right\rangle$. Multiplying Eq. (46) by $P_{q}$ and then integrating over $P_{q}$, we get the evolution equation for $\left\langle P_{q}\right\rangle$,

$$
\frac{\partial\left\langle P_{q}\right\rangle}{\partial \ln r}=-2 b \widetilde{T}(q)\left\langle P_{q}\right\rangle,
$$

with

$$
\begin{aligned}
\widetilde{T}(q) & =\frac{1}{\pi} \int_{0}^{\pi / 2} \frac{d \theta}{\sin ^{2} \theta \cos ^{2} \theta}\left(1-\cos ^{2 q} \theta-\sin ^{2 q} \theta\right) \\
& =\frac{2}{\sqrt{\pi}} \frac{\Gamma(q-1 / 2)}{\Gamma(q-1)} \\
& =\frac{1}{2^{2 q-3}} \frac{\Gamma(2 q-1)}{\Gamma(q) \Gamma(q-1)} .
\end{aligned}
$$

We assumed here that $q>\frac{1}{2}$, which is the condition of the existence of the integral in Eq. (48). For smaller $q$ the resonance approximation breaks down. Integrating Eq. (47) from $r=1$ to $r \sim N$, we find the multifractal behavior $\left\langle P_{q}\right\rangle$ $\sim N^{-\tilde{\tau}(q)}$ with the exponents

$$
\tilde{\tau}(q)=2 b \widetilde{T}(q) .
$$

The function $\widetilde{T}(q)$ is shown in Fig. 5. Its asymptotics are

$$
\begin{gathered}
\widetilde{T}(q) \simeq-\frac{1}{\pi(q-1 / 2)}, \quad q \rightarrow 1 / 2 ; \\
\widetilde{T}(q) \simeq \frac{2}{\sqrt{\pi}} q^{1 / 2}, \quad q \gg 1 .
\end{gathered}
$$

We see that the fractal exponents are proportional to the small parambeter $b$. This is characteristic of wave functions

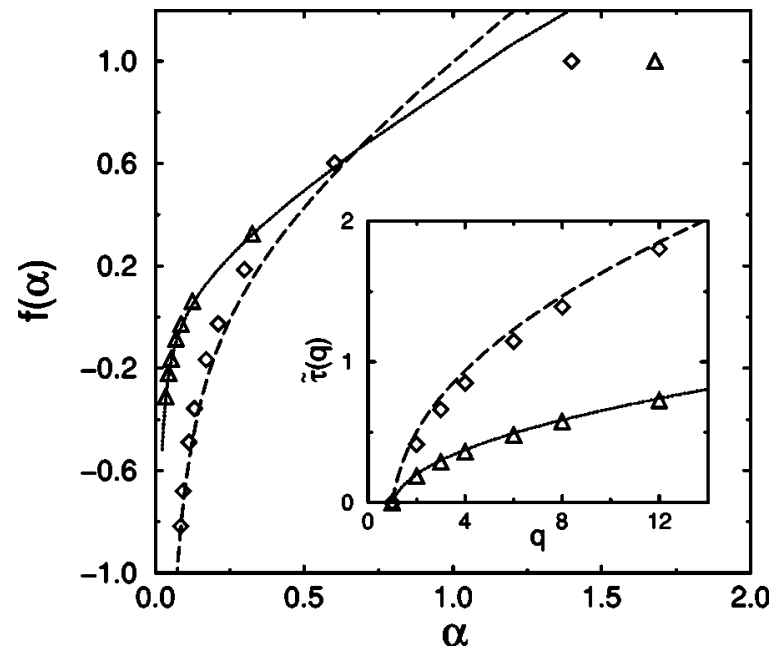

FIG. 6. Multifractal spectrum $f(\alpha)$ for $b=0.25(\diamond)$ and $b$ $=0.1(\triangle)$. Inset: exponent $\tilde{\tau}(q)$. Dashed and dotted lines indicate the analytical results Eqs. (52) and (49).

that are very small, typically, with rare and strong peaks (resonances). In the limit $b \rightarrow 0$ the fractal exponents tend to their insulator value $\tilde{\tau}(q)=0$.

Legendre transformation of Eq. (49) produces the $f(\alpha)$ spectrum of the form

$$
f(\alpha)=2 b F(A), \quad A=\alpha / 2 b,
$$

where $F(A)$ is the Legendre transform of $\widetilde{T}(q)$. The function $F(A)$ is shown in the inset of Fig. 5; its asymptotics are

$$
\begin{aligned}
& F(A) \simeq-\frac{1}{\pi A}, \quad A \rightarrow 0 ; \\
& F(A) \simeq \frac{A}{2}, \quad A \rightarrow \infty .
\end{aligned}
$$

Furthermore, it changes sign at $A_{-} \simeq 0.5104$, corresponding to $q_{c} \simeq 2.4056$. These analytical findings are fully supported by numerical simulations as can be seen from Fig. 6 .

We return now to the IPR distribution function. The scale invariance of the limiting distribution has been already demonstrated via the numerical solution of Eq. (46); see Fig. 4. To show this also analytically, we make the ansatz

$$
f\left(P_{q}, r\right)=r^{\tau(q)} f_{0}\left(P_{q} r^{\tau(q)}\right) .
$$

Equation (55) is just the statement of $r$ independence of the limiting distribution $\mathcal{P}\left(P_{q} / P_{q}^{\mathrm{typ}}\right)$, with $P_{q}^{\mathrm{typ}}$ scaling as $P_{q}^{\mathrm{typ}}$ $\propto r^{-\tau(q)}$. Substituting Eq. (55) into Eq. (46), we get the equation

$$
\begin{aligned}
\tau(q)\left[f_{0}\left(\widetilde{P}_{q}\right)+\widetilde{P}_{q} f_{0}^{\prime}\left(\widetilde{P}_{q}\right)\right] \\
=\frac{2 b}{\pi} \int_{0}^{\pi / 2} \frac{d \theta}{\sin ^{2} \theta \cos ^{2} \theta} \\
\quad \times\left(-f_{0}\left(\widetilde{P}_{q}\right)+\int d \widetilde{P}_{q}^{(1)} d \widetilde{P}_{q}^{(2)} f_{0}\left(\widetilde{P}_{q}^{(1)}\right) f_{0}\left(\widetilde{P}_{q}^{(2)}\right)\right. \\
\left.\quad \times \delta\left(\widetilde{P}_{q}-\widetilde{P}_{q}^{(1)} \cos ^{2 q} \theta-\widetilde{P}_{q}^{(2)} \sin ^{2 q} \theta\right)\right)
\end{aligned}
$$


The fact that the scale $r$ has dropped out from Eq. (56) implies the consistency of the ansatz (55) for the fixed-point distribution. To demonstrate the significance of this statement, we make a more general ansatz for the limiting distribution,

$$
f\left(P_{q}, r\right)=\frac{1}{P_{q} \sigma(r)} g_{0}\left(\frac{\ln \left(P_{q} r^{\tau(q)}\right)}{\sigma(r)}\right),
$$

which allows for a change of the width of the distribution of $\ln P_{q}$ with $r$, characterized by a function $\sigma(r)$ [note that $\sigma(r)$ is defined up to a constant factor, which can be absorbed into the definition of the function $g_{0}$ ]. At $\sigma(r)=1$ this reduces to our earlier ansatz (45), while at $\sigma(r)=\ln r$ we get the form proposed in Ref. 19. Substituting Eq. (57) in Eq. (46), we find that the $r$ dependence drops out of the resulting equation for $g_{0}$ if and only if $\sigma(r)=$ const. This means an inconsistency of the ansatz (57) with a nonconstant $\sigma(r)$ and, in particular, excludes the possibility of a universal distribution of fractal exponents $[\sigma(r)=\ln r]$ advocated in Ref. 19.

We turn now to the power-law tail of this scale-invariant distribution, $f_{0}\left(\widetilde{P}_{q}\right) \sim \widetilde{P}_{q}^{-x_{q}-1}$. In order to calculate the index $x_{q}$, we consider Eq. (56) in the limit $\widetilde{P}_{q} \gg 1$. It is easy to see that the integral $\int d \widetilde{P}_{q}^{(1)} d \widetilde{P}_{q}^{(2)} \ldots$ in the right-hand side of Eq. (56) is dominated by the region $\widetilde{P}_{q}^{(1)} \sim \widetilde{P}_{q}, \widetilde{P}_{q}^{(2)} \sim 1$ (or vice versa), the contribution of the region $\widetilde{P}_{q}^{(1)} \sim \widetilde{P}_{q}^{(2)} \sim \widetilde{P}_{q}$ being suppressed by an additional factor of $\widetilde{P}_{q}^{-x_{q}}$. Furthermore, when $\widetilde{P}_{q}^{(1)} \sim \widetilde{P}_{q}$ and $\widetilde{P}_{q}^{(2)} \sim 1$, we can neglect $\widetilde{P}_{q}^{(2)}$ in the argument of the $\delta$ function. The integrals over $\widetilde{P}_{q}^{(1)}$ and $\widetilde{P}_{q}^{(2)}$ then become trivial, and Eq. (56) reduces to

$$
\tau(q) x_{q}=\frac{2 b}{\pi} \int_{0}^{\pi / 2} \frac{d \theta}{\sin ^{2} \theta \cos ^{2} \theta}\left(1-\sin ^{2 q x} q \theta-\cos ^{2 q x} q \theta\right) .
$$

Comparing this with Eqs. (48) and (49), we see that the right-hand side of Eq. (58) is nothing but $\tilde{\tau}\left(q x_{q}\right)$, so that Eq. (58) can be rewritten in the form (16).

We analyze now Eq. (16) in the regimes $q<q_{c}$ and $q$ $>q_{c}$. In the case $q<q_{c}$ we expect $x_{q}>1$ and $\tau(q)=\tilde{\tau}(q)$. The latter statement can be directly proven by applying the operation $\int d \widetilde{P}_{q} \widetilde{P}_{q} \cdots$ to Eq. (56). [Clearly, this proof breaks down for $q>q_{c}$ because of the divergence of the integral $\int d \widetilde{P}_{q} \widetilde{P}_{q} f_{0}\left(\widetilde{P}_{q}\right)$.] Graphical interpretation of Eq. (16) for $q$ $<q_{c}$ is shown in Fig. 7; its solution $x_{q}>1$ decreases with increasing $q$, reaching unity at $q=q_{c}$, as expected. For the most frequently studied case $q=2$ ("conventional" IPR) we find $x_{2}=\frac{3}{2}$. As to the $q>q_{c}$ regime, we have then $\tau(q)$ $=q \alpha_{-}$, and the solution of Eq. (16) has a very simple form,

$$
x_{q}=\frac{q_{c}}{q}, \quad q>q_{c} .
$$

Let us remind the reader that up to now we considered in this subsection the ensemble of real matrices $(\beta=1)$. However, all the above considerations are also applicable to the case $\beta=2$, with a minor modification. Specifically, the measure of the $V$ integration in Eq. (43) should be modified:

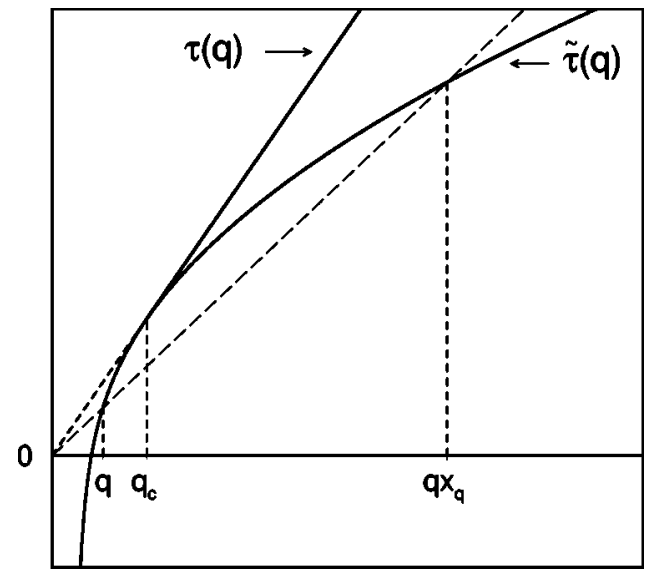

FIG. 7. Graphical interpretation of Eq. (16) for $q<q_{c}$.

$$
\int_{-\infty}^{\infty} d V \frac{\tilde{r}}{b \sqrt{2 \pi}} e^{-V^{2} \tilde{r}^{2} / 2 b^{2}} \cdots \rightarrow \int_{0}^{\infty} d V V \frac{2 \widetilde{r}^{2}}{b^{2}} e^{-V^{2} \tilde{r}^{2} / b^{2}} \cdots
$$

This leads, after the $V$ integration, to the replacement of $b$ by $(\pi / 2 \sqrt{2}) b$. With this substitution, all results of this subsection remain valid for $\beta=2$.

\section{Finite-size effects in the scaling of the IPR distribution}

Since in reality one always has to deal with systems of a finite size, the understanding of finite-size effects is important for an accurate interpretation of numerical data. In Fig. 8 we show the evolution of the distribution $\mathcal{P}\left(\ln P_{2}\right)$ with $N$ for three values of $b$, representative of the small- $b$, the large- $b$, and the crossover regimes.

In the case $b \ll 1$, the evolution of the IPR distribution with $N$ is governed by the "time" $t=b \ln N$, so that $t \gg 1$ is the condition of the proximity to the fixed point. Therefore, at small $b$ one needs exponentially large values of $N$ in order to reach the limiting distribution. [Note that this is not true for the average $\left\langle P_{q}\right\rangle$, the evolution of which is governed by Eq. (47), implying a much weaker condition $N \gg 1$ for the scaling regime.] The logarithmically slow approach to the limiting distribution is clearly seen in Figs. 8(c) and 9.

At $b \gg 1$ the convergence to the fixed-point distribution is much faster [Fig. 8(b)]. In this regime the condition for the scaling of the IPR distribution is $N^{1 / 2} / b \gg 1$, as can be seen by comparing the relative magnitude of the IPR fluctuations at the critical point, $\left[\operatorname{var}\left(P_{2}\right)\right]^{1 / 2} /\left\langle P_{2}\right\rangle \simeq 0.41 / b$ [see Eq. (26)], with that in the Gaussian orthogonal ensemble, $\left[\operatorname{var}\left(P_{2}\right)\right]^{1 / 2} /\left\langle P_{2}\right\rangle \simeq 1.64 / N^{1 / 2}$. Extrapolating the numerically found values of the relative variance to $1 / N=0$, we find good agreement with the theoretical prediction (see Fig. 10). Note a qualitative difference in the approach to the fixed-point distribution at small and large $b$ : while at $b \ll 1$ the height of the distribution $\mathcal{P}\left(\ln P_{2}\right)$ decreases with $N$, approaching the limiting value from above, the behavior is opposite at $b \gg 1$.

Extrapolating the small- $b$ and large- $b$ results to the crossover range $b \sim 1$, we find simply the condition $N \gg 1$ for the proximity to the fixed-point distribution. Therefore, in the crossover regime $b \sim 1$ the limiting distribution can be reached most easily. This is precisely what we observe in our numerical simulations. Figure 8(a), representing the evolu- 

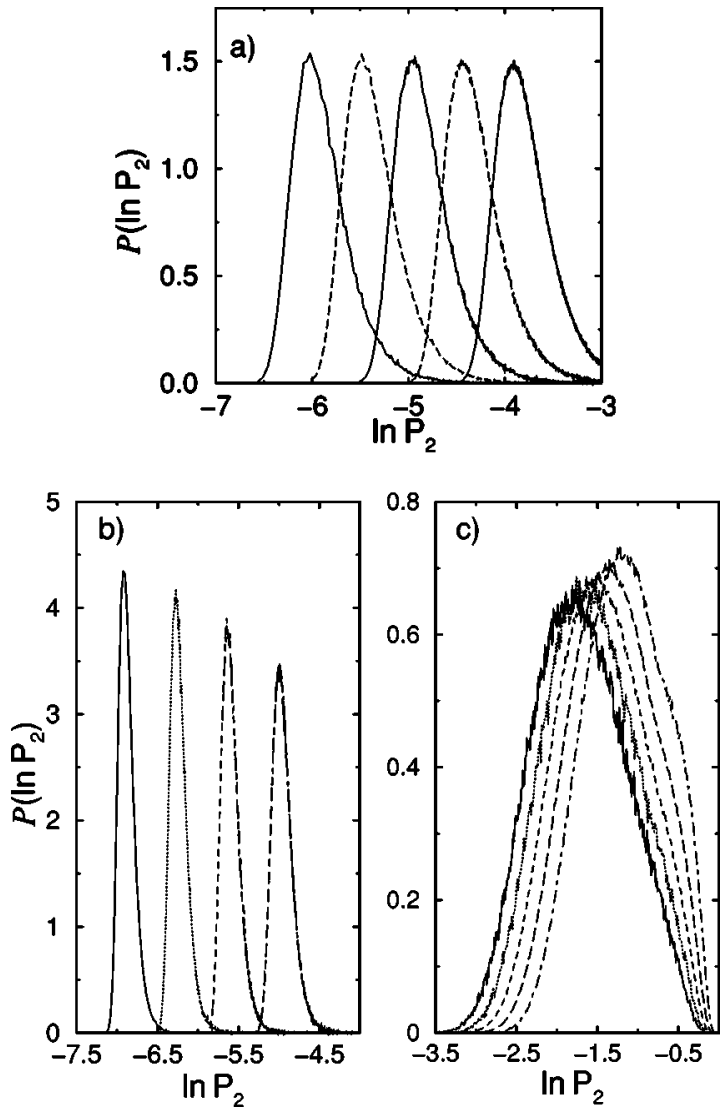

FIG. 8. Evolution of the distribution $\mathcal{P}\left(\ln P_{2}\right)$ for (a) $b=1$, (b) $b=4$, and (c) $b=0.1$ with the system size $N$ [from left to right: $N$ $=4096,2048,1024,512(256)]$.

tion of the IPR distribution at $b=1$, demonstrates the almost perfect scale invariance of the distribution with only very small deviations (less than $3 \%$ ) over a range of system sizes from $N=256 \cdots 4096$.

Let us also comment on other types of "finite-size effects" that appear in numerical simulations. Numerically, it is impossible to perform an average at precisely a given value of the energy. Instead, one averages over an energy interval $\Delta E$ that one would like to choose as big as possible in order to improve statistics. On the other hand, it is clear that $\Delta E$ should not be too big in order to avoid mixing of

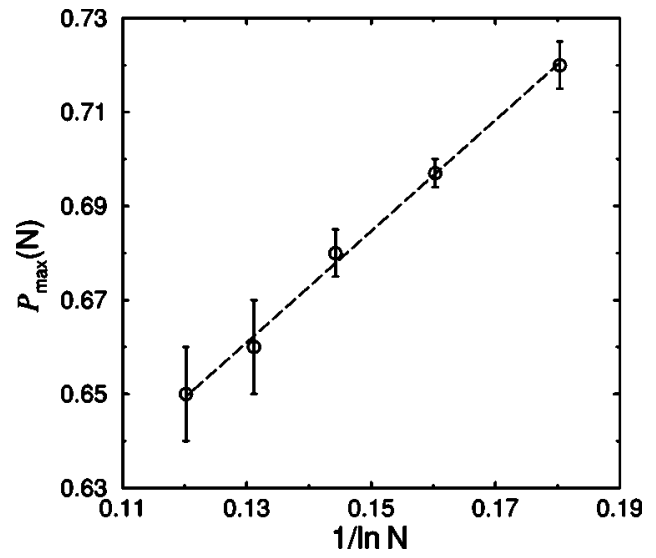

FIG. 9. Maximum value of $\mathcal{P}\left(\ln P_{2}\right)$ for $b=0.1$ as a function of the system size. Extrapolation to $1 / \ln N=0$ yields $\mathcal{P}_{\max }(\infty) \simeq 0.51$.

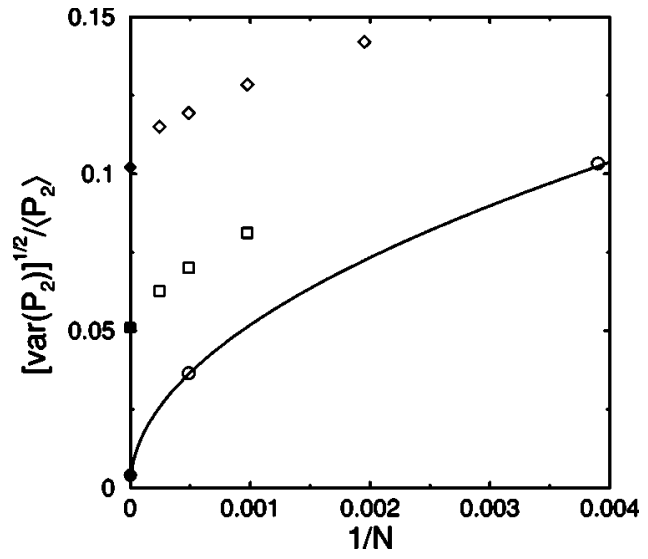

FIG. 10. Variance of $P_{2}$ in the limit of large system sizes $N$ for $b=100(\bigcirc), b=8(\square)$, and $b=4(\diamond)$. The filled symbols denote the theoretical result $(26)$ valid at $1 \ll b \ll \sqrt{N}$, the solid line indicates the RMT limit, $b \gg \sqrt{N}$, where $\left[\operatorname{var}\left(P_{2}\right)\right]^{1 / 2} /\left\langle P_{2}\right\rangle \approx 1.64 / \sqrt{N}$.

different critical theories. In our simulations we have chosen $\Delta E$ to be about $10 \%$ of the bandwidth. This value is still small enough, the corresponding variation of the density of states $\nu(E)$ being of the order of $1 \%$.

Furthermore, the size $S$ of the matrix ensemble over which the average was taken is an important parameter in the simulations. Typical values we have used are $N=256$, $S=30000 ; \quad N=512, \quad S=10000 ; \quad N=1024, \quad S=5000 ; N$ $=2048, S=1000 ; N=4096, S=100$. In some cases, like for the two-level correlation function $R_{2}(s)$ or the full IPR distribution function, the ensemble has to be much larger. In these cases, we give the numbers explicitly in the figure caption.

\section{LEVEL STATISTICS}

The two-level correlation function is defined in the usual way,

$$
R_{2}(\omega)=\frac{1}{\langle\nu(E)\rangle^{2}}\langle\nu(E+\omega / 2) \nu(E-\omega / 2)\rangle
$$

where $\nu(E)=N^{-1} \operatorname{Tr} \delta(E-\hat{H})$ is the fluctuating density of states. At the critical point $R_{2}(\omega)$ acquires a scale-invariant form [if considered as a function of $s=\omega / \Delta$, the frequency normalized to the mean level spacing $\Delta=1 / N\langle\nu(E)\rangle] \cdot{ }^{30-32}$ The distinct feature of the critical level statistics is a nontrivial value of the spectral compressibility $0<\chi<1$ characterizing the linear behavior of the variance of the number $n(\mathcal{E})$ of levels in an energy window $\mathcal{E}^{30,33,34}$

$$
\operatorname{var}[n(\mathcal{E})]=\chi\langle n(\mathcal{E})\rangle, \quad\langle n(\mathcal{E})\rangle \equiv \frac{\mathcal{E}}{\Delta} \gg 1
$$

The compressibility $\chi$ can be expressed through the connected part $R_{2}^{(c)}(s)=R_{2}(s)-1$ of the critical level correlation function as follows:

$$
\chi=\int_{-\infty}^{\infty} d s R_{2}^{(c)}(s)
$$


Recently, it was argued in Ref. 35 that Eq. (7) constitutes an exact relation between the spectral compressibility $\chi$ and the fractal dimension $D_{2}$. The derivation of Eq. (7) in Ref. 35 is based on Dyson's idea of Brownian motion through the ensemble of Hamiltonians combined with some assumption of the decoupling of the energy level and wave-function correlations previously proposed in Ref. 39 . While this decoupling has been proven to work up to three-loop order in the $1 / g$ expansion in $2 \mathrm{D},{ }^{39}$ its applicability in the strongcoupling regime remained in the status of a conjecture. The PRBM model allows us to check the validity of the relation (7). Similarly to the IPR distribution function, the level correlation function can be calculated analytically in the two limits $b \gg 1$ and $b \ll 1$ and numerically in the full range of $b$.

In the $b \gg 1$ regime, the two-level correlation function is obtained by an appropriate generalization of the earlier findings for the diffusive samples; ${ }^{40,41}$ the results can be found in Refs. 20, 42, and 15. In particular, considering for simplicity the $\beta=2$ ensemble at the band center, the level correlation function has the form

$$
R_{2}^{(c)}(s)=\delta(s)-\frac{\sin ^{2}(\pi s)}{(\pi s)^{2}} \frac{(\pi s / 4 b)^{2}}{\sinh ^{2}(\pi s / 4 b)} .
$$

The correlation function (64) follows the RMT result $R_{2}^{(c)}(s)=\delta(s)-\sin ^{2}(\pi s) /(\pi s)^{2}$ up to the scale $s \sim b$ (playing the role of the Thouless energy here), and then begins to decay exponentially. The spectral compressibility at $b \gg 1$ is given by ${ }^{20,15}$

$$
\chi \simeq \frac{1}{2 \pi \beta b}, \quad b \gg 1 .
$$

Comparing this with Eq. (23), one finds ${ }^{20}$ that the formula (7) is indeed satisfied to leading order in $1 / b$.

We now turn to the opposite limit $b \ll 1$. The evolution equation for $R_{2}(\omega, r)$ can be written down in analogy with Eq. (43):

$$
\begin{aligned}
\frac{\partial R_{2}(\omega, r)}{\partial r}= & \frac{2}{N} \int_{-\infty}^{\infty} d \tau \int_{-\infty}^{\infty} d V \frac{1}{\sqrt{2 \pi}} \frac{\tilde{r}}{b} e^{-V^{2} \tilde{r}^{2} / 2 b^{2}} 2|V| \\
& \times\left[\delta\left(\omega-2 V \sqrt{1+\tau^{2}}\right)-\delta(\omega-2 V \tau)\right] .
\end{aligned}
$$

Equation (66) should be integrated over $r$ from $r=0$ to $N / 2$ with the boundary condition $R_{2}(\omega, 0)=1$; the result $R_{2}(\omega, N / 2)$ will then give the desired level correlation function. Evaluating the $V$ integral in Eq. (66) and changing the variables to $z=2 r / N$ and $x=2 V / \omega$, we get at $s=\omega / \Delta>0$

$$
\begin{aligned}
R_{2}^{(c)}(s)= & -1+\int_{0}^{1} d z \frac{s}{\pi b} \sin \frac{\pi z}{2} \int_{0}^{1} \frac{d x}{\sqrt{1-x^{2}}} \\
& \times \exp \left(-\frac{s^{2} x^{2}}{4 \pi b^{2}} \sin ^{2} \frac{\pi z}{2}\right) .
\end{aligned}
$$

After some algebra, we find the level correlation function to be given by

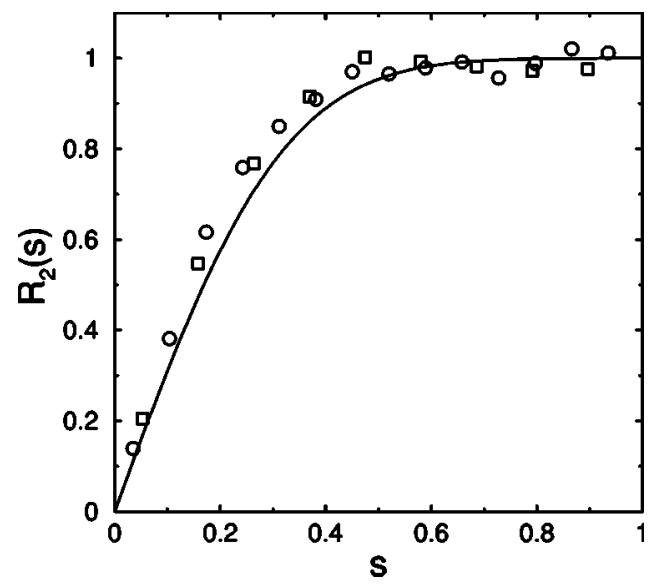

FIG. 11. Two-level correlation function $R_{2}(s)$ for two system sizes $N=256(\bigcirc)$ and $N=512(\square)$ at $b=0.1$. The solid line indicates the theoretical result (68). Number $S$ of matrices in the ensemble: $N=256, S=3440512 ; N=512, S=1418688$.

$$
R_{2}^{(c)}(s)=\delta(s)-\operatorname{erfc}\left(\frac{|s|}{2 \sqrt{\pi} b}\right),
$$

where $\operatorname{erfc}(x)=(2 / \sqrt{\pi}) \int_{x}^{\infty} \exp \left(-t^{2}\right) d t$ is the error function and we have included the $\delta(s)$ contribution due to the selfcorrelation of the energy levels. Substitution of Eq. (68) in Eq. (63) yields the spectral compressibility

$$
\chi \simeq 1-4 b, \quad b \ll 1 .
$$

We see, therefore, that in the limit of small $b$ the level repulsion is efficient in a narrow region $|s| \lesssim b$ only, and the spectral compressibility tends to the Poisson value $\chi=1$. The physical reason for the reduced range of the level repulsion is quite transparent. Consider two states nearby in energy separated by a typical distance $r \sim N$ in the coordinate space. If their energy difference $s \lesssim b$, two such states will form a resonance pair, so that their levels will repel. On the other hand, if $s \gg b$, these two states will not be in resonance, their wave functions remain weakly overlapping, and the level repulsion between them will be inefficient.

Hence, formula (7), which would predict $\chi \rightarrow \frac{1}{2}$ at $b \rightarrow 0$, is violated. Similar violation of Eq. (7) is indicated by numerical data for the tight-binding model in dimensions $d$ $\geqslant 4 .{ }^{43}$ Most likely it is never an exact relation, but rather an approximation valid in the weak-multifractality limit only. Strictly speaking, our results do not rule out the possibility that Eq. (7) is exact at $b$ exceeding a certain value $b_{c}$ and breaks down at $b<b_{c}$. However, we do not see a physical reason for such a qualitative change being induced by the variation of $b$.

These results are fully supported by our numerical data. In particular, Fig. 11 represents the level correlation function $R_{2}(s)$ at $b=0.1$ showing a nice agreement with Eq. (68). Note that the finite-size effects in the level correlation function at $b \ll 1$ are much weaker than in the IPR distribution (Sec. II D). Indeed, the only assumption (besides $b \ll 1$ ) used in the derivation of the evolution equation (66) is $N \gg 1$, and then Eq. (68) is obtained without any further approximations. Therefore, in contrast to the IPR statistics, which reaches its fixed-point form at exponentially large $N$ (the condition be- 


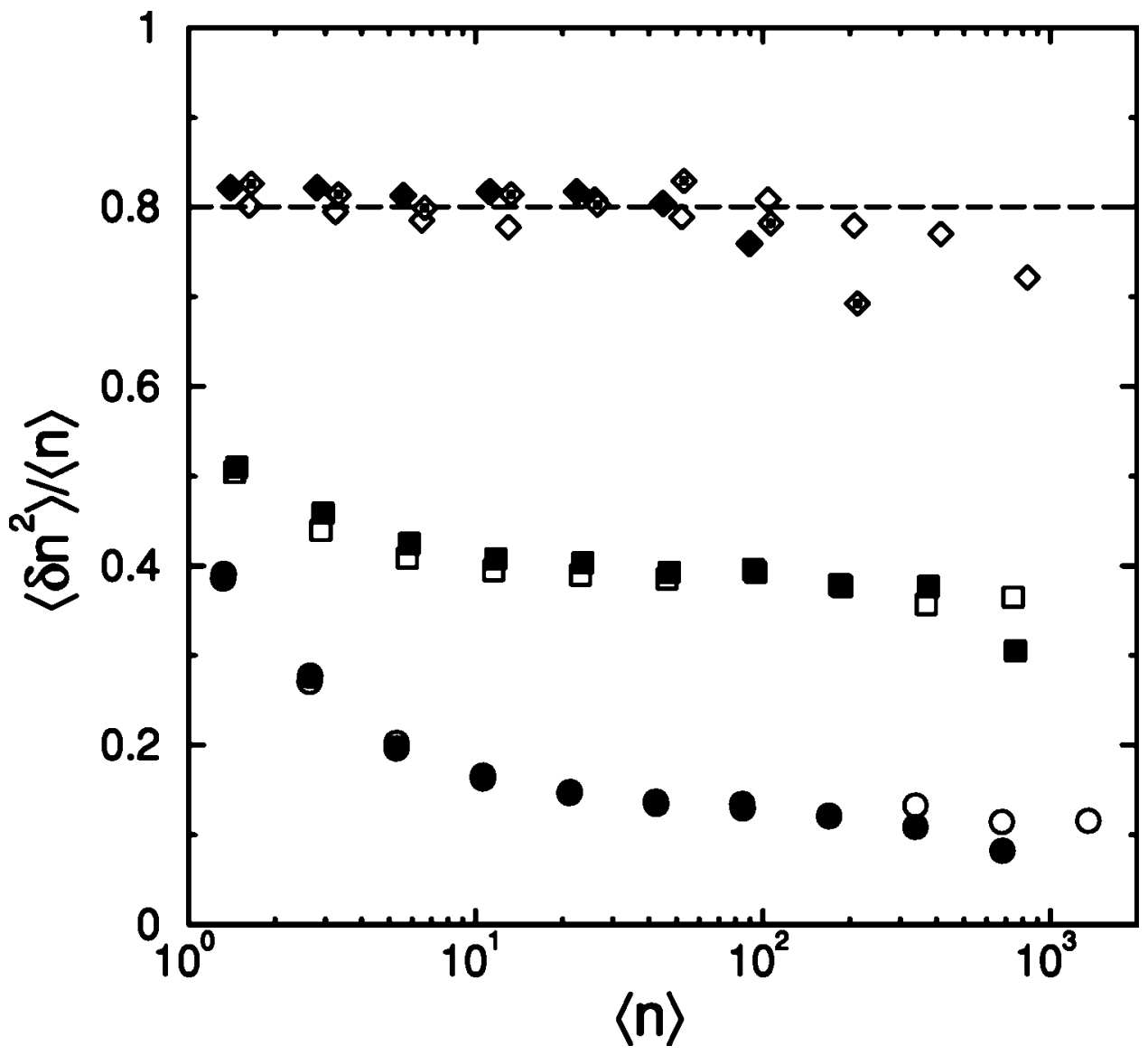

FIG. 12. Variance of the number of levels $\left\langle\delta n^{2}\right\rangle$ in a fixed energy interval as a function of the energy width of the interval parametrized by the mean level number $\langle n\rangle$ it contains. Traces correspond to $b=1$ (open $\bigcirc: N=4096$, filled: $N=2048$ ), $b=0.25$ (open $\square: N=4096$, filled: $N=2048$ ), and $b=0.05$ (open $\diamond: N=4096$, $\diamond$ with dot: $N=1024$, filled: $N=512$ ). Statistical errors are typically of the order of the symbol size. The dashed line indicates the analytical prediction Eq. (69), for $b=0.05$.

ing $b \ln N \gg 1$ ), the level statistics acquires the $N$-invariant form already at $N \gg 1$, see Figs. 11 and 12 . At $b \gg 1$, the fixed-point condition is $N \gg b^{2}$ (the same as for the IPR distribution, see Sec. II D).

To find numerically the spectral compressibility $\chi$, we plot the level number variance $\operatorname{var}[n(\mathcal{E})]$ versus the average $\langle n(\mathcal{E})\rangle$ (Fig. 12). The data show an extended plateau region in $\operatorname{var}[n(\mathcal{E})] /\langle n(\mathcal{E})\rangle$, determining $\chi$. The upper bound for this region is set by the matrix size $N$, while the lower bound is $\sim b$ [the value of the upper limit at which the integral (63) saturates]. We see that the data traces are independent of the system size $N$ (with exception of the large- $\langle n\rangle$ cutoff determined by $N$ ) within the statistical errors. The numerically obtained spectral compressibility in the broad range of $b$ is shown in Fig. 13; in the large- $b$ and small- $b$ regions it agrees well with the corresponding analytical asymptotics.

The above calculation is easily modified to the $\beta=2$ case, by replacing the measure of the $V$ integration in Eq. (66) according to Eq. (60). Performing the $V$ integral, we now get

$$
\begin{aligned}
R_{2}^{(c)}(s)= & -1+\int_{0}^{1} d z \frac{s^{2}}{\pi b^{2}} \sin ^{2} \frac{\pi z}{2} \int_{0}^{1} d u \\
& \times \exp \left[-\left(1-u^{2}\right) \frac{s^{2}}{2 \pi b^{2}} \sin ^{2} \frac{\pi z}{2}\right],
\end{aligned}
$$

which yields the result

$$
R_{2}^{(c)}(s)=\delta(s)-\exp \left(-\frac{s^{2}}{2 \pi b^{2}}\right)
$$

The spectral compressibility is thus equal to

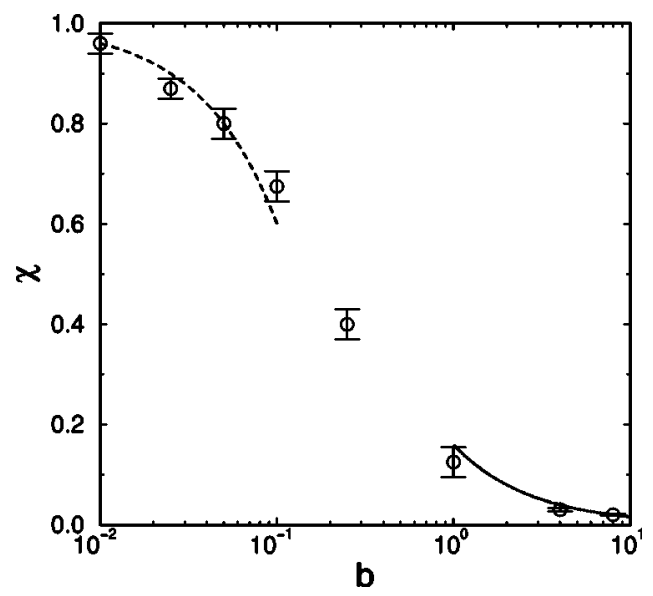

FIG. 13. Spectral compressibility $\chi$ as a function of $b$ : crossover from the "quasimetallic" ( $b \gg 1)$ to the "quasi-insulating" ( $b$ $\ll 1$ ) behavior. The lines indicate the analytical results for $b \gg 1$ and $b \ll 1$, Eqs. (65) and (69). 


$$
\chi \simeq 1-\pi \sqrt{2} b .
$$

Again we see that the region of level repulsion shrinks in the limit $b \rightarrow 0$, with the compressibility approaching the Poisson value $\chi=1$.

It is worth mentioning that the above results for the case $\beta=2$ can also be obtained by exploiting the relation ${ }^{42,15}$ between the PRBM model and a random matrix ensemble introduced by Moshe, Neuberger, and Shapiro. ${ }^{44}$ This mapping, which becomes exact in both limits $b \ll 1$ and $b \gg 1$, relates the level statistics (but not the eigenfunction statistics) of the two ensembles. On the other hand, the level correlation function of the ensemble of Moshe et al. can be calculated exactly in the case $\beta=2$ and is in fact identical to the density correlation function of a 1D noninteracting Fermi gas at a finite temperature. ${ }^{44}$ Applying the results of Ref. 44, one obtains for the $\beta=2$ PRBM ensemble precisely the results (64) and (65) at $b \gg 1$ (Refs. 42 and 15) and (71) and (72) at $b \ll 1 .{ }^{15}$

\section{CONCLUSIONS}

In this paper, we have presented a detailed study of the statistics of eigenfunctions and energy levels in the family of the critical PRBM models. We have obtained analytical results for the IPR distribution function, the multifractal spectrum, and the level correlation function in the two limits of weak and strong multifractality $(b \gg 1$ and $b \ll 1)$, by using the supersymmetry approach ${ }^{15}$ and the resonance renormalization-group method, ${ }^{28,38}$ respectively. The analytical results are fully supported by numerical simulations, which also have allowed us to explore the crossover region $(b \sim 1)$.

Our main findings can be summarized as follows.

(i) The distribution function of the IPR (normalized to its typical value $P_{q}^{\text {typ }}$ ) is scale-invariant in the limit of large system size $N$. In other words, the distribution function of the IPR logarithm, $\mathcal{P}\left(\ln P_{q}\right)$, shifts along the $x$ axis with increasing $N$, without changing its form and width. At $b \gg 1$ and at not too large $q \ll(2 \pi \beta b)^{1 / 2}$, the "body" of the distribution is found to have a simple analytical form (30). In the opposite limit $b \ll 1$ the distribution function is given by the solution of the integro-differential equation (56).

(ii) The scaling of $P_{q}^{\text {typ }}$ with the system size, $P_{q}^{\text {typ }}$ $\propto N^{-D_{q}(q-1)}$, defines the fractal exponent $D_{q}$, which is a nonfluctuating quantity, in contrast to Ref. 19.

(iii) The scale-invariant distribution $\mathcal{P}\left(z \equiv P_{q} / P_{q}^{\text {typ }}\right)$ has a power-law tail $\propto z^{-1-x_{q}}$. At sufficiently large $q>q_{c}(b)$ one finds $x_{q}<1$, and the average value $\left\langle P_{q}\right\rangle \propto N^{-\tilde{D}_{q}(q-1)}$ becomes nonrepresentative and scales with a different exponent $\widetilde{D}_{q} \neq D_{q}$. The "phase boundary" separating the regimes of $x_{q}>1\left(\widetilde{D}_{q}=D_{q}\right)$ and $x_{q}<1 \quad\left(\widetilde{D}_{q} \neq D_{q}\right)$ has the asymptotics $q_{c}=(2 \pi \beta b)^{1 / 2}$ at $b \gg 1$ and $q_{c} \simeq 2.41$ at $b \ll 1$.

(iv) The singularity spectrum $f(\alpha)$ obtained from $\tilde{\tau}(q)$ $\equiv \widetilde{D}_{q}(q-1)$ via the Legendre transformation has a close-toparabolic form (24) in the weak-multifractality regime $b$ $\gg 1$ and is given by Eqs. (52)-(54) in the opposite limit $b$ $\ll 1$. The point $\alpha_{-}$of the singularity spectrum determined by the condition $f\left(\alpha_{-}\right)=0$ corresponds to the IPR selfaveraging boundary $q_{c}$ given above.

(v) The critical spectral statistics shows a crossover from a "quasimetallic"' (close-to-RMT) behavior (64) at $b \gg 1$ to a "quasi-insulating", (close-to-Poisson) one, Eqs. (68) and (71), at $b \ll 1$. In particular, the spectral compressibility changes from 0 to 1 , thus violating the relation (7) (argued to be exact in Ref. 35) in the strong-multifractality regime.

(vi) Finally, we note that the precise form of both the IPR distribution and the level correlation function depends on the behavior of the hopping amplitude $a(r)$ of the PRBM model at distances of the order of the system size, $r \sim N$. This is analogous to the dependence of these distributions on the sample shape and the boundary conditions in the critical point of the Anderson transition in $d$ dimensions. ${ }^{46}$ We have used the form (6) of $a(r)$ playing a role of the periodic boundary conditions for PRBM. On the other hand, the critical exponents $D_{q}, \widetilde{D}_{q}$, and $x_{q}$, as well as the singularity spectrum $f(\alpha)$ and the spectral compressibility $\chi$, are independent of this choice and determined solely by the relevant critical theory.

Using the analogy discussed in the Introduction, we expect that these results are qualitatively valid also for the conventional Anderson transition in $d$ dimensions, with $d=2$ $+\epsilon$ and $d \gg 1$ playing the role of the weak- and strongcoupling limits, respectively. However, a fair amount of analytical and numerical work is still needed to verify this conjecture. In fact, recent (partly unpublished as yet) numerical results on the critical level statistics in $d=4,5,6$ (Ref. 43) suggest that the critical statistics tend to the Poisson limit (with the spectral compressibility $\chi \rightarrow 1$ ) as $d \rightarrow \infty$, in full agreement with our results for the PRBM ensemble. On the other hand, the only available numerical study of the IPR distribution at criticality in 3D (Ref. 19) led the authors to the conclusion of strong (scale-invariant) fluctuations of the fractal dimension $D_{2}$, in stark contrast with our findings. We believe that the reason is in too small system sizes and a not sufficiently careful analysis of the numerical data in Ref. 19. Clearly, additional work in this direction is needed. Analytical study of statistical properties of the wave functions and energy levels at criticality in the $d \gg 1$ limit also remains a challenge for future research.

\section{ACKNOWLEDGMENTS}

Discussions with Y.V. Fyodorov, V.E. Kravtsov, L.S. Levitov, D.G. Polyakov, I. Varga, and I.Kh. Zharekeshev are gratefully acknowledged. This work was supported by the SFB 195 der Deutschen Forschungsgemeinschaft.
*Also at Petersburg Nuclear Physics Institute, 188350 St. Petersburg, Russia.

${ }^{1}$ F. Wegner, Phys. Rep. 67, 15 (1980).

${ }^{2}$ K.B. Efetov, Adv. Phys. 32, 53 (1983); Supersymmetry in Disor- der and Chaos (Cambridge University Press, Cambridge, 1997).

${ }^{3}$ F. Wegner, Z. Phys. B 36, 209 (1980).

${ }^{4}$ C. Castellani and L. Peliti, J. Phys. A 19, L429 (1986).

${ }^{5}$ B.B. Mandelbrot, J. Fluid Mech. 62, 331 (1974). 
${ }^{6}$ G. Paladin and A. Vulpiani, Phys. Rep. 156, 147 (1987).

${ }^{7}$ C. Mudry, C. Chamon, and X.-G. Wen, Phys. Rev. Lett. 77, 4194 (1996); Nucl. Phys. B 466, 383 (1996).

${ }^{8}$ H.E. Castillo, C. Chamon, E. Fradkin, P.M. Goldbart, and C. Mudry, Phys. Rev. B 56, 10668 (1997).

${ }^{9}$ J.-S. Caux, N. Taniguchi, and A.M. Tsvelik, Phys. Rev. Lett. 80, 1276 (1998); Nucl. Phys. B 525, 671 (1998); J.-S. Caux, Phys. Rev. Lett. 81, 4196 (1998).

${ }^{10}$ M. Janssen, M. Metzler, and M.R. Zirnbauer, Phys. Rev. B 59, 15836 (1999).

${ }^{11}$ M.J. Bhaseen, I.I. Kogan, O.A. Soloviev, N. Taniguchi, and A.M. Tsvelik, Nucl. Phys. B 580, 688 (2000).

${ }^{12}$ M. Janssen, Int. J. Mod. Phys. B 8, 943 (1994); Phys. Rep. 295, 1 (1998).

${ }^{13}$ B. Huckestein, Rev. Mod. Phys. 67, 357 (1995).

${ }^{14}$ J.T. Chalker and G.J. Daniell, Phys. Rev. Lett. 61, 593 (1988); J.T. Chalker, Physica A 167, 253 (1990).

${ }^{15}$ A.D. Mirlin, Phys. Rep. 326, 259 (2000).

${ }^{16}$ Y.V. Fyodorov and A.D. Mirlin, Phys. Rev. B 51, 13403 (1995).

${ }^{17}$ V.N. Prigodin and B.L. Altshuler, Phys. Rev. Lett. 80, 1944 (1998).

${ }^{18}$ B. Shapiro, Phys. Rev. B 34, 4394 (1986).

${ }^{19}$ D.A. Parshin and H.R. Schober, Phys. Rev. Lett. 83, 4590 (1999).

${ }^{20}$ A.D. Mirlin, Y.V. Fyodorov, F.-M. Dittes, J. Quezada, and T.H. Seligman, Phys. Rev. E 54, 3221 (1996).

${ }^{21}$ J.V. José and R. Cordery, Phys. Rev. Lett. 56, 290 (1986).

${ }^{22}$ A.V. Balatsky and M.I. Salkola, Phys. Rev. Lett. 76, 2386 (1996).

${ }^{23}$ B.L. Altshuler and L.S. Levitov, Phys. Rep. 288, 487 (1997).

${ }^{24}$ I.V. Ponomarev and P.G. Silvestrov, Phys. Rev. B 56, 3742 (1997).

${ }^{25}$ G. Casati and T. Prosen, Physica D 131, 293 (1999); F. Borgonovi, P. Conti, D. Rebuzzi, B. Hu, and B. Li, ibid. 131, 317 (1999).

${ }^{26}$ V.E. Kravtsov, Ann. Phys. (Leipzig) 8, 621 (1999); V.E. Kravtsov and A.M. Tsvelik, cond-mat/0002120(unpublished).

${ }^{27} \mathrm{We}$ concentrate on the band center $E=0$. Changing the energy $E$ at fixed $b$ also allows us to go through the one-parametric family of the critical points; this way is, however, numerically inconvenient.

${ }^{28}$ L.S. Levitov, Phys. Rev. Lett. 64, 547 (1990).

${ }^{29}$ M.R. Zirnbauer, hep-th/9905054(unpublished).

${ }^{30}$ B.L. Altshuler, I.K. Zharekeshev, S.A. Kotochigova, and B.I. Shklovskii, Zh. Éksp. Teor. Fiz. 94, 343 (1988) [Sov. Phys. JETP 67, 625 (1988)].

${ }^{31}$ B.I. Shklovskii, B. Shapiro, B.R. Sears, P. Lambrianides, and H.B. Shore, Phys. Rev. B 47, 11487 (1993).

${ }^{32}$ V.E. Kravtsov, I.V. Lerner, B.L. Altshuler, and A.G. Aronov, Phys. Rev. Lett. 72, 888 (1994).

${ }^{33}$ A.G. Aronov and A.D. Mirlin, Phys. Rev. B 51, 6131 (1995).

${ }^{34}$ V.E. Kravtsov and I.V. Lerner, Phys. Rev. Lett. 74, 2563 (1995).

${ }^{35}$ J.T. Chalker, V.E. Kravtsov, and I.V. Lerner, Pis'ma Zh. Éksp. Teor. Fiz. 64, 355 (1996) [JETP Lett. 64, 386 (1996)].

${ }^{36}$ F. Evers and A.D. Mirlin, Phys. Rev. Lett. 84, 3690 (2000).

${ }^{37}$ V.I. Fal'ko and K.B. Efetov, Europhys. Lett. 32, 627 (1995); Phys. Rev. B 52, 17413 (1995).

${ }^{38}$ L.S. Levitov, Ann. Phys. (Leipzig) 8, 697 (1999).

${ }^{39}$ J.T. Chalker, I.V. Lerner, and R.A. Smith, Phys. Rev. Lett. 77, 554 (1996); J. Math. Phys. 37, 5061 (1996).

${ }^{40}$ V.E. Kravtsov and A.D. Mirlin, Pis'ma Zh. Éksp. Teor. Fiz. 60, 645 (1994) [JETP Lett. 60, 656 (1994)].

${ }^{41}$ A.V. Andreev and B.L. Altshuler, Phys. Rev. Lett. 75, 902 (1995).

${ }^{42}$ V.E. Kravtsov and K.A. Muttalib, Phys. Rev. Lett. 79, 1913 (1997).

${ }^{43}$ I. Kh. Zharekeshev and B. Kramer, Ann. Phys. (Leipzig) 7, 442 (1998); I. Kh. Zharekeshev (unpublished).

${ }^{44}$ M. Moshe, H. Neuberger, and B. Shapiro, Phys. Rev. Lett. 73, 1497 (1994).

${ }^{45}$ I. Varga and D. Braun, Phys. Rev. B 61, R11 859 (2000).

${ }^{46}$ For numerical study of the dependence of the critical level statistics on the sample geometry in 3D, see D. Braun, G. Montambaux, and M. Pascaud, Phys. Rev. Lett. 81, 1062 (1998); H. Potempa and L. Schweitzer, J. Phys.: Condens. Matter 10, L431 (1998). 